\title{
DO SOCIO-DEMOGRAPHIC AND CORONA REASSURANCE- SEEKING BEHAVIOUR PREDICT CORONA ANXIETY? - A STUDY AMONG INDIAN POPULATION
}

\author{
Debaraj Das' ${ }^{1}$, Suchitra Pall \\ 1. School of Human Resource Management, XIM University, Bhubaneswar, India \\ Correspondence: debaraj9@gmail.com
}

\begin{abstract}
INTRODUCTION:

The COVID-19 pandemic has caused severe anxiety and affected mental and psychological health of the people. Virus related anxiety is thought to be affected by several socio-demographic factors, individual's risk-perception, and personality.

\section{OBJECTIVE:}

The objective of the study was to investigate the predictors of corona virus-related general anxiety and death anxiety during the COVID-19 outbreak in India. More specifically, the study examined the COVID-19 related reassurance-seeking behaviours, neuroticism, health anxiety, and risk perception among the adult population.

\section{DESIGN:}

A survey methodology was adopted to collect data from the general adult population ( $N=550$ ) in India between October 29th to November 10th, 2020. Data analysis was carried out using multiple regression analysis in SPSS to examine the significant predictors of general anxiety and death anxiety of the participants. The analysis used the incremental validity of Corona reassurance-seeking behaviours in the multiple regression model.
\end{abstract}

\section{SETTING:}

The study was conducted among the Indian general population.

\section{FINDINGS:}

Major significant predictors of general anxiety related pandemic included demographic factors like being female, being young, and less educated. Simultaneously, we observed common predictors of virus-related general anxiety and death anxiety, Corona reassurance-seeking behaviours, health anxiety, and neuroticism. The participant's risk perception predicted the participant's death anxiety but not the general anxiety during the pandemic.

\section{DISCUSSION:}

The present study's findings will help the policymakers handle pandemic related stress in a better manner.

\section{KEYWORDS}

COVID-19; risk perception; anxiety; death anxiety; neuroticism; corona reassurance-seeking behaviours 
Life has not been the same ever since the World Health Organisation (WHO) declared Coronavirus (SARS-COV-2; COVID-19) as a pandemic in March 2020. India soon figured among the top three most affected countries in the world causing chaos in every sphere of life existence. Highrisk perception [1] of the pandemic created aggravated fear [2,3] - better coined as 'Coronaphobia' [4,5], anxiety [5-7], and emotional distress [8] among all sections of the population affecting physical and psychological health and well-being.

Fear is a negative emotion and is an adaptive defense mechanism, and excessive fear is associated with an anxiety disorder [3]. The fear of the virus infection arising out of perceived risk with the pandemic, disruptions to life, work and economy, social isolation, and loneliness are creating a 'perfect storm' for emotional distress [8] and a breeding ground for psychological consequences. [9] The COVID -19 fear is associated with increased anxiety, aggravated depression, thoughts of suicide, hopelessness, and other psychological consequences. [5,6] Besides, the relatively high mortality rate of the COVID-19 pandemic compared to the previous pandemics is also found to cause death anxiety among individuals. [5]

Individuals who are vulnerable to negative emotions (neuroticism) and health anxiety, exhibit higher emotional distress. [5,10] Individuals with higher vulnerability also perceive symptoms of common flu and illness associated with COVID-19 and tend to seek reassurance [5] from friends, relatives, doctors, medical staff, and self-checking to relieve their anxiety. Hence, perceived risk associated with the pandemic related vulnerability factors of neuroticism, health anxiety, and reassurance seeking behaviour may predict coronavirus anxiety and death anxiety among general populations.

While examining Italian community-dwelling adults demographic characteristics on perceived emotional problems due to COVID-19, Somma et al. observed that females and age significantly contributed to the clinically relevant emotional issues. [11] Similarly, young female adults reported a higher risk perception than males in a study of emotional distress during the pandemic on young adults in Zurich, Switzerland. [12] In a previous study of risk perception among European and Asian countries during SARS flu influenza, de Zwart et al. observed that Asia perceived a higher vulnerability than Europe. [13] They found that comparative vulnerability was lower in women than in men, but higher among less educated. In a study during the 2009 Influenza $A(\mathrm{H} 1 \mathrm{Nl})$ pandemic in the Netherlands, Bults et al. observed that with an increase in perceived risk and high vulnerability, people with higher age, and higher anxiety took to preventive measures. [14] Hence socio-demographic factors such as gender, age, education level, and family type may determine individuals' risk perception and affect psychological wellbeing. However, past studies indicate mixed results. Exploring this in the collectivistic Indian society may throw new lights.

Negative emotions and feelings during a pandemic continue for a long time affecting long-term psychological well-being. While there have been observations of behavioural changes among people [15], there are reported observations of anxiety and stress disorders [2,3,5$7,16]$ during COVID-19 pandemic. In a recent study in Poland, $50.1 \%$ reported high to very high perceived-risk, and $58.8 \%$ believed the long-term consequences of COVID-19 to be severe to very severe. [17]

While Lee et al.'s study focused on the population in the USA, the rationale of this study was to explore the effect of risk perception and vulnerability factors on corona anxiety and death anxiety in India. More specifically, the objectives of this paper are: 1) to examine the socio-demographic impacts on corona anxiety and death anxiety during the COVID-19, 2) to explore the effect of risk-perception, neuroticism, health anxiety, and reassurance seeking behaviours on corona anxiety and death anxiety, and 3) to explore corona anxiety exclusively in India when the COVID-19 infection in India was at its peak. Lee investigated the cognitive, behavioural, emotional, and psychological aspects of COVID-19 and validated Corona Anxiety Scale (CAS) for research use. [6] We, therefore, used CAS in the Indian context. We believe that perceived risk and associated fear, anxiety, and death anxiety might throw new light into emotional well-being and their effect on participants' demographics, age, gender, education, and family type. These findings may help in designing specific measures to address the people's psychological needs by the service providers. 


\section{PARTICIPANTS AND PROCEDURE}

We have collected the data in the first week of November 2020 on a pan India basis. A convenience sample of 550, out of which 346 males and 204 females participated in this study. The mean age of the participants was 37 years and $S D=0.98$; range 18-66. The participants were required to give their consent and had to be 18 years of age. We used the participants' socio-demographic factors like gender, age, education background, and family type (joint or nuclear family). We did not consider employment status as we believed industry specific job might have different outcomes. Instead, we focussed on the general population. Most of the participants are living in joint families (69\%), had earned at least a bachelor's degree or above (73\%), and did know someone with COVID-19 (64\%).

\section{MEASURES}

For operationalisation of the construct vulnerability factors, we considered the pandemic related three vulnerability factors described by Taylor in his book "The Psychology of Pandemics". [10] The personality trait of neuroticism is considered as the variable depicting the vulnerability of individuals having tendency for negative emotions and is measured with the eight items scale developed by John and Srivastava. [18] The participants rated their responses on a five-point Likert scale, where 1 stands for 'disagree strongly' to 5 for 'agree strongly.' One example of the scale's survey items includes, 'I see myself as someone who gets nervous easily.' The Cronbach's alpha for this scale is .70.

The second vulnerability factor, health anxiety (worrying too much about health) of participants during COVID-19 was measured using the Patient Health Questionnaire (PHQ 9) developed by Kroenke et al. [19]. It is a self-administered version of the Prime MD, e.g., 'over the last two weeks, how often have you bothered by feeling down, depressed or hopeless.' Each of these nine questionnaires of PHQ was rated as ' 0 ' (not at all) to ' 3 ' (nearly every day). The internal consistency of this PHQ 9 is .90.

The third vulnerability factor, Coronavirus reassuranceseeking behaviours (CRSB) was measured using the Lee et al.'s scale [5]. CRSB was developed by Lee et al. to measure the tendency of individuals seeking reassurance that they are not infected with the virus. It is a five-point Likert scale where 0 = 'not at all' to $4=$ 'nearly every day for the last two weeks'. The participants need to express how often they experience each activity in the previous two weeks, a sample questionnaire, e.g., 'I spoke with other people about my symptoms to see if I was infected with the coronavirus disease.' The scale reliability was .85.

We used Dryhurst et al.'s six items risk perception scale specially designed to measure the Coronavirus/COVID-19 risk. [1] It is a self-assessment scale measured on a sevenpoint Likert-scale ranging from 1 = 'not at all worried' to $7=$ 'very worried.' Out of the six items, four items measured risk perceiving towards self, and two items measured risk perceiving towards others. An example item includes: 'How worried are you personally about the following issues at present Coronavirus/COVID-19?' The internal consistency (Cronbach's alpha) for the scale was .73.

Corona Anxiety was measured using Lee's five-items Corona Anxiety Scale (CAS), where participants rated on a 0 (not at all) to 4 (nearly every day over the last two weeks) scale [6]. One of the scale items, for example, includes, 'I felt dizzy, lightheaded, or faint when I read or listened to news about the coronavirus'. The internal consistency of the scale was .85. The second outcome measure 'death anxiety' was measured using Abdel-Khalek's single item death anxiety scale [20], the statement of the scale 'I am afraid of death'. Participants rated the extent to which they agreed on a 1 (disagree strongly) to 5 (agree strongly) scale.

\section{RESULTS}

\section{DESCRIPTIVE STATISTICS AND PARTIAL CORRELATION}

The descriptive statistics reveal that a significant number of participants experienced Corona anxiety and death anxiety. The partial correlation demonstrated that corona reassurance-seeking behaviour was significantly associated with age $(r=-.16)$, education $(r=.18)$, family type $(r=-.09)$, COVID-19 factors $(r=-.17)$, risk perception ( $r$ $=.29)$, neuroticism ( $r=.29)$, physical health $(r=0.52)$, corona anxiety ( $r=0.59)$, and corona related death anxiety $(r=.37)$ (Refer Table 1). The socio-demographic variables, COVID19 factors, vulnerability factors, and outcomes variables were also intercorrelated. We included all these variables in the regression analysis. 


\begin{tabular}{lcccccccc}
\hline VARIABLES & MEAN & SD & CAS & CRSB $\begin{array}{l}\text { RISK } \\
\text { PERCEPION }\end{array}$ & NEUROTICISM & $\begin{array}{l}\text { HEALTH } \\
\text { ANXIETY }\end{array}$ & $\begin{array}{l}\text { DEATH } \\
\text { ANXIETY }\end{array}$ \\
\hline CAS & .52 & .71 & - & $.59^{* *} .22^{* *}$ & $.41^{* *}$ & $.62^{* *}$ & $.40^{* *}$ \\
CRSB & .85 & .88 & $.59^{* *}$ & - & $.29^{* *}$ & $.29^{* *}$ & $.52^{* *}$ & $.37^{* *}$ \\
Risk perception & 4.07 & 1.06 & $.22^{* *}$ & $.29^{* *}-$ & $.31^{* *}$ & $.24^{* *}$ & $.43^{* *}$ \\
Neuroticism & 2.57 & .81 & $.41^{* *}$ & $.29^{* *}$ & $.31^{* *}$ & - & $.52^{* *}$ & $.41^{* *}$ \\
Health anxiety & .55 & .65 & $.62^{* *}$ & $.52^{* *}$ & $.24^{* *}$ & $.52^{* *}$ & - & $.41^{* *}$ \\
Death anxiety & 2.80 & 1.56 & $.40^{* *}$ & $.37^{* *}$ & $.43^{* *}$ & $.41^{* *}$ & $.41^{* *}$ & - \\
\hline
\end{tabular}

Notes: CAS: Corona Anxiety Scale; CRSB: Coronavirus Reassurance Seeking Behaviours.

** Correlation is significant at the 0.01 level (2-tailed); $N=550$

\section{INDEPENDENT SAMPLE T-TEST}

\section{Gender: Men vs. Women}

Independent samples t-test compared the general anxiety measures between men and women. There was a significant difference in Corona anxiety scores between men and women, $\dagger(550)=2.45, p<.01$. Specifically, these results suggest that women report more significant anxiety than men, even in the time of the COVID-19. But we found no gender difference in death anxiety among the participants.

\section{Family: Joint vs. nuclear family}

The second independent sample t-test compared CAS scores and death anxiety scores between participants from a joint family and nuclear family, ( $\dagger(550)=1.41, p=N S)$ and $(\dagger(550)=1.42, p=N S)$. There was no significant statistical difference in the Corona anxiety and corona death anxiety in participants from the joint and nuclear family.

\section{Education: Graduate vs. less than graduate}

The third independent sample t-test compared between CAS scores and participants educational background it $(550)=-3.89, p<.001)$. It gives a clear picture that less educated people (participants with an education level less than a graduate) have higher corona anxiety. There is no difference in death anxiety $(t(546)=3.69, p=N S)$ among graduate or less than graduate participants.

We took the first one-way ANOVA to find out the role of age in CAS, and we found a statistically significant difference between the age of the participants $(F(3,546)=3.96, p<$
$.001)$. A Tukey post hoc test clearly shows that participants of 25 years or less have a significant difference than participants in the age bracket of 41 to 60 years in their CAS $(p<.01)$. There was no statistically significant difference between the other age groups in their CAS scores. We also found there is a significant difference in death anxiety scores of different age groups $(F(3,546)=4.65, p<.01)$.

\section{HIERARCHICAL MULTIPLE REGRESSION ANALYSIS}

After screening the data, we found no issue with singularity, multicollinearity, the dependence of errors, normality, linearity, or homoscedasticity as suggested by Tabachnick et al. [21] We used the Hunsley and Meyer's approach for two separate multiple regression analyses. [22] For further analysis of the present study, we used risk perception and the vulnerability factors of neuroticism, health anxiety, and CRSB as the predictor variables and corona anxiety and death anxiety as outcome variables (see Table 2 and Figure 1). We used the stepwise regression method in the first step of the regression analysis included the sociodemographic variables age, gender $(1=$ female, $2=$ male, 3 = others.), education ( $1=$ graduate and above, $2=$ less than a graduate), and family type $(1=$ joint family, $2=$ nuclear family). The second step added the COVID-19 factors like personal knowledge of someone with COVID19 or personally diagnosed ( 1 = yes, 2 = no). In the third step, we have added psychological distress during a pandemic: neuroticism, health anxiety, and risk perception related to COVID-19 pandemic. In the fourth step, we used corona reassurance-seeking behaviours. The outcome variables are corona anxiety and corona death anxiety (see Table 2 for regression summary). 
In the first regression analysis, Corona anxiety was the outcome variable. In the first step, age $(\beta=-.09, p<.05)$ and formal education $(\beta=.13, p<.01)$ as the sociodemographic variables that were significant predictors in the model, $R^{2}=.04, F(4,544)=6.27, p<.001$. In the second step, when the COVID-19 factor is included, again age $(\beta$ $=-.09, p<.05)$ and formal education $(\beta=.12, p<.01)$ were significant predictor variables $\left(R^{2}=.04, F(5,543)=5.04, p<\right.$ $.001)$. In the third step, when the vulnerability factors were included, neuroticism $(\beta=0.10, p<.01)$, health anxiety $(\beta=$ $.54, p<.001)$, and risk perception $(\beta=.07, p<.05)$ were significant in the model $\left(R^{2}=.40, F(8,540)=45.33, p<.001\right)$.
In the final step, when corona reassurance seeking behaviours was included, 'knowledge of someone with COVID-19' $(\beta=.06, p<.05)$, neuroticism $(\beta=0.11, p<.01)$, health anxiety $(\beta=.37, p<.001)$, and corona reassurance seeking behaviours $(\beta=.38, p<.001)$ were the only significant predictor variables in the model $\left(R^{2}=.50, F(9\right.$, $539)=59.91, p<.001)$. These results support the incremental validity of corona reassurance seeking behaviours in explaining $10 \%$ more variance in corona anxiety symptoms above socio-demographics, COVID-19 factors, and vulnerability factors.

\section{TABLE 2: HIERARCHICAL MULTIPLE REGRESSION}

\begin{tabular}{|c|c|c|c|c|}
\hline \multirow{2}{*}{ PREDICTORS } & \multicolumn{2}{|c|}{ CORONA ANXIETY } & \multicolumn{2}{|c|}{ DEATH ANXIETY } \\
\hline & BETA & $\mathbf{T}$ & BETA & $\mathbf{T}$ \\
\hline Gender & -.01 & -.38 & .05 & 1.30 \\
\hline Age & .06 & 1.74 & .03 & .80 \\
\hline Education & .01 & .37 & .07 & 1.86 \\
\hline Family type & .01 & .38 & -.01 & -.44 \\
\hline $\begin{array}{l}\text { Do you know someone previously } \\
\text { with COVID-19 }\end{array}$ & .06 & $1.98^{*}$ & .09 & $2.69^{* *}$ \\
\hline Risk perception & .00 & .13 & .32 & $8.15^{* * *}$ \\
\hline Neuroticism & .11 & $3.09^{* *}$ & .20 & $4.69^{* * *}$ \\
\hline Health anxiety & .37 & $9.22^{* * *}$ & .15 & $3.33^{* * *}$ \\
\hline CRSB & .38 & $10.29^{* * *}$ & .14 & $3.41^{* * *}$ \\
\hline $\mathbf{R}^{2}$ & .50 & & .34 & \\
\hline Change in $\mathbf{R}^{2}$ & .10 & & .01 & \\
\hline Significant F Change & $\mathrm{p}<.001$ & & $p<.001$ & \\
\hline
\end{tabular}

Notes: CRSB: Coronavirus Reassurance Seeking Behaviours.

* $\mathrm{p}<.05 ;{ }^{* *} \mathrm{p}<.01 ;{ }^{* * *} \mathrm{p}<.001$

In the second regression analysis death anxiety was the outcome variable. In the first step age $(\beta=-.13, p<.01)$ and formal education $(\beta=.08, p<.05)$ as the socio demographic variable that were significant predictor in the model $\left(R^{2}=.03, F(4,544)=4.90, p<.001\right)$. In the second step when the COVID-19 factor (e.g., knowledge of someone with COVID-19) was included again age $(\beta=-.13$, $p<.01)$ and formal education $(\beta=.08, p<.05)$ were both significant predictor variables $\left(R^{2}=.03, F(5,543)=4.19, p<\right.$
$.001)$. In the third step, when the vulnerability factors were included, COVID-19 factor ( $\beta=.08, p<.05)$, neuroticism ( $\beta$ $=.20, p<.001)$, health anxiety $(\beta=.22, p<.001)$, and risk perception $(\beta=.34, p<.001)$ were significant in the model $\left(R^{2}=.32, F(8,540)=32.67, p<.001\right)$. In the final step, when vulnerability factor of CSRB was included, COVID-19 factor $(\beta=.09, p<.01)$, neuroticism $(\beta=0.20, p<.001)$, health anxiety $(\beta=.15, p<.001)$, risk perception $(\beta=.32, p<.001)$, and coronavirus reassurance seeking behaviours $(\beta=.14, p$ $<.001$ ) were the significant predictor variables in the model 
$\left(R^{2}=.34, F(9,539)=30.90, p<.001\right)$. These results modestly support the incremental validity of corona reassurance seeking behaviours in explaining $1.4 \%$ more variance in death anxiety symptoms above socio-demographics, COVID-19 factors, and vulnerability factors.

\section{FIGURE 1: EFFECT OF PREDICTORS ON OUTCOME VARIABLES}

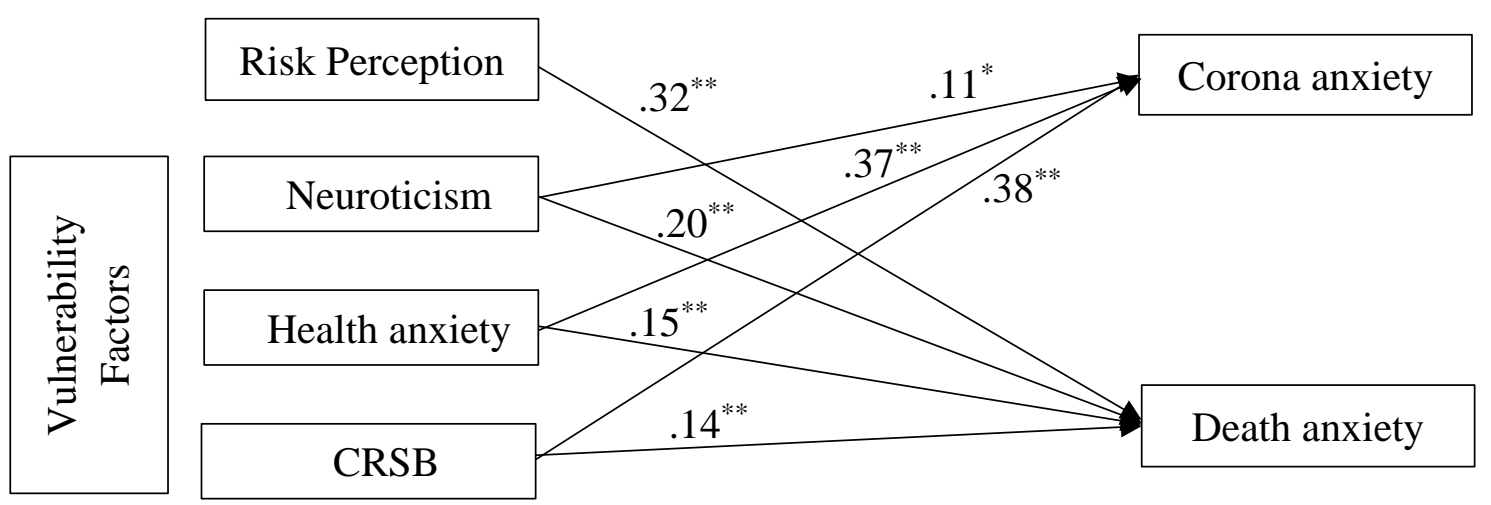

Notes: CRSB: Coronavirus Reassurance Seeking Behaviours.

$* p<.01 ; * * p<.001$. Only significant coefficients are shown.

\section{DISCUSSION}

The purpose of the study was to find the predictors of corona anxiety and death anxiety during the COVID-19 pandemic in India. The result of two multiple regression analyses suggests that Corona reassurance-seeking behaviours, health anxiety, and neuroticism are the common predictors of corona anxiety and death anxiety during the COVID-19 pandemic in India. From the independent sample t-test it is clear that the level of CAS among women respondents are higher than the male respondents during the COVID-19 pandemic, which is supported by previous studies [23-26]. Younger and less educated group of participants are experiencing higher anxiety, which is also supported by the previous work of Taylor et al. [25]

Health anxiety is a function of how likely and how severe society perceives the illness. [27] This study is unique in its finding that the health-related anxiety concern during the pandemic and neuroticism are significant predictors of both Corona anxiety and death anxiety, which is alien based on the previous research findings. $[24,28]$ First used in this pandemic, the Corona reassurance-seeking behaviour was a significant predictor of Corona anxiety and death anxiety among Indian respondents, which contradicts the result of Lee et al. [5,25] studied in western settings.

Next, in the current study, participants' risk perception towards COVID-19 predicts the participants' death anxiety but not corona anxiety. People overestimate the risk of adverse outcomes due to excessive emotional stress. To our knowledge, this was the first time the risk perception scale of Dryhurst et al. was tested among Indian participants and found as a reliable measure of risk perception during the COVID-19 pandemic.

Past research and media reports show that old age increases the COVID-19 related infection and mortality rate. In our study, the younger participants have higher COVID-19 general anxiety and death anxiety than the older respondents, supported by an Iranian research. [29] The implication of the findings of this study is that the policy makers may plan effective proactive measures to mitigate the anxiety, mental, and emotional stress among Indian population during pandemic situations.

Few limitations need to be considered before interpreting the result. Before interpreting the results, we must consider the limitations. First, these data are cross-sectional and solely based on the self-reported online responses, which 
may not be free from the socially desirable responses. Future research may work on longitudinal data related to a pandemic to understand corona anxiety and death anxiety. Second, we used convenience sampling for data collection due to the ongoing pandemic situation. Therefore, replication and extension of this research may overcome by using a more diverse sample. Third, we have studied the health anxiety of the participants without knowing their previous long-term health issues. The participant's pre-COVID-19 health issues may influence their health anxiety responses.

\section{CONCLUSIONS}

This study used corona reassurance-seeking behaviours by Lee et al. [5] and risk perception in India's collectivistic culture. In a collectivistic society, group harmony and group relationship play a significant role in social interactions. Future research should examine whether culture plays a role in responding to a pandemic. This study is unique in the Indian context to explore the incremental validity analysis of the corona reassurance-seeking behaviours construct developed and validated in the United States of America. The study provides significant findings which can be used by policy makes and healthcare providers to plan effective measures to address people's psychological aspects during a pandemic.

\section{References}

1. Dryhurst S, Schneider CR, Kerr J, Freeman ALJ, Recchia G, van der Bles AM, Spiegelhalter D, van der Linden S. Risk perceptions of COVID-19 around the world. J Risk Res. 2020;1-13.

2. Harper CA, Satchell LP, Fido D, Latzman RD. Functional Fear Predicts Public Health Compliance in the COVID19 Pandemic. Int J Ment Health Addict [Internet]. 2020 [cited 2020 Aug 3]; Available from: http://link.springer.com/10.1007/s 1 1469-020-00281-5

3. Ornell F, Schuch JB, Sordi AO, Kessler FHP. "Pandemic fear" and COVID-19: mental health burden and strategies. Braz J Psychiatry. 2020;42(3):232-5.

4. Asmundson GJG, Taylor S. Coronaphobia: Fear and the 2019-nCoV outbreak. J Anxiety Disord. 2020;70:102196.
5. Lee SA, Jobe MC, Mathis AA, Gibbons JA. Incrementa validity of coronaphobia: Coronavirus anxiety explains depression, generalized anxiety, and death anxiety. J Anxiety Disord. 2020;74:102268.

6. Lee SA. Coronavirus Anxiety Scale: A brief mental health screener for COVID-19 related anxiety. Death Stud. 2020;44(7):393-401.

7. Wang $C$, Pan R, Wan X, Tan Y, Xu L, Mclntyre RS, Choo FN, Tran B, Ho R, Sharma VK, Ho C. A longitudinal study on the mental health of general population during the COVID-19 epidemic in China. Brain Behav Immun. 2020;87:40.

8. Shanahan L, Steinhoff A, Bechtiger L, Murray AL, Nivette A, Hepp U, Ribeaud D, Eisner M. Emotional distress in young adults during the COVID-19 pandemic: evidence of risk and resilience from a longitudinal cohort study. Psychol Med. 2020;1-10.

9. Aslam F, Awan TM, Syed JH, Kashif A, Parveen M. Sentiments and emotions evoked by news headlines of coronavirus disease (COVID-19) outbreak. Humanit Soc Sci Commun. 2020;9.

10. Taylor S. The psychology of pandemics: Preparing for the next global outbreak of infectious disease. 2019 ed. Newcastle upon Tyne: Cambridge Scholars Publishing; 2019.

11. Somma A, Gialdi G, Krueger RF, Markon KE, Frau C, Lovallo S, Fossati A. Dysfunctional personality features, non-scientifically supported causal beliefs, and emotional problems during the first month of the COVID-19 pandemic in Italy. Personal Individ Differ. 2020;165:110139.

12. Shanahan L, Steinhoff A, Bechtiger L, Murray AL, Nivette A, Hepp U, Ribeaud D, Eisner M. Emotional distress in young adults during the COVID-19 pandemic: evidence of risk and resilience from a longitudinal cohort study. Psychol Med. 2020;1-10.

13. de Zwart O, Veldhuijzen IK, Elam G, Aro AR, Abraham T, Bishop GD, Voeten HACM, Richardus JH, Brug J. Perceived Threat, Risk Perception, and Efficacy Beliefs Related to SARS and Other (Emerging) Infectious Diseases: Results of an International Survey. Int J Behav Med. 2009;16(1):30-40.

14. Bults M, Beaujean DJ, de Zwart O, Kok G, van Empelen $P$, van Steenbergen JE, Richardus JH, Voeten HA. Perceived risk, anxiety, and behavioural responses of the general public during the early phase of the Influenza A ( $\mathrm{H} 1 \mathrm{~N} 1)$ pandemic in the Netherlands: 
results of three consecutive online surveys. BMC Public Health. 2011;11(1):2.

15. Khosravi M. Perceived Risk of COVID-19 Pandemic: The Role of Public Worry and Trust. Electron J Gen Med. 2020;17(4):em203.

16. Roy D, Tripathy S, Kar SK, Sharma N, Verma SK, Kaushal V. Study of knowledge, attitude, anxiety \& perceived mental healthcare need in Indian population during COVID-19 pandemic. Asian J Psychiatry. 2020;51:102083.

17. Malesza M, Kaczmarek MC. Predictors of anxiety during the COVID-19 pandemic in Poland. Personal Individ Differ. 202;170:1 10419.

18. John OP, Srivastava S. Big Five Personality TaxonomyThe big five trait taxonomy: history, measurement, and theoretical perspectives. In: Handbook of Personality theory and research. Guilford Press; 1999. p. 102-38. (In L. A. Pervin, \& O. P. John (Eds.)).

19. Kroenke K, Spitzer RL, Williams JBW. Validity of a Brief Depression Severity Measure. J Gen Intern Med. $2001 ; 16: 606-13$

20. Abdel-Khalek AM. Single versus multi-item scales in measuring death anxiety. Death Stud. 2010;22(8):76372.

21. Tabachnick BG, Fidell LS, Ullman JB. Using multivariate statistics. Seventh edition. NY, NY: Pearson; 2019. 832 p.

22. Hunsley J, Meyer GJ. The Incremental Validity of Psychological Testing and Assessment: Conceptual, Methodological, and Statistical Issues. Psychol Assess. 2003; 15(4):446-55

23. Egloff B, Schmukle SC. Gender differences in implicit and explicit anxiety measures. Personal Individ Differ. 2004;36(8):1807-15.

24. Maaravi Y, Heller B. Not all worries were created equal: the case of COVID-19 anxiety. Public Health. 2020;185:243-5.

25. Taylor MR, Agho KE, Stevens GJ, Raphael B. Factors influencing psychological distress during a disease epidemic: Data from Australia's first outbreak of equine influenza. BMC Public Health. 2008;8(1):347.

26. Wang C, Pan R, Wan X, Tan Y, Xu L, Ho CS, Ho RC. Immediate Psychological Responses and Associated Factors during the Initial Stage of the 2019 Coronavirus Disease (COVID-19) Epidemic among the General Population in China. Int J Environ Res Public Health. 2020;17(5):1729.
27. Wheaton MG, Abramowitz JS, Berman NC, Fabricant LE, Olatunji BO. Psychological Predictors of Anxiety in Response to the HINl (Swine Flu) Pandemic. Cogn Ther Res. 2012;36(3):210-8.

28. Anagnostopoulos F, Botse T. Exploring the Role of Neuroticism and Insecure Attachment in Health Anxiety, Safety-Seeking Behavior Engagement, and Medical Services Utilization: A Study Based on an Extended Interpersonal Model of Health Anxiety. SAGE Open. 2016;6(2):1-13.

29. Moghanibashi-Mansourieh A. Assessing the anxiety level of Iranian general population during COVID-19 outbreak. Asian J Psychiatry. 2020;51:102076. 